\title{
An Equivalent Circuit Modeling of an Equispaced Metallic Nanoparticles (MNPs) Plasmon Wire
}

\author{
Kyungjun Song and Pinaki Mazumder, Fellow, IEEE
}

\begin{abstract}
Based on the electric dipole moment (EDM) model of free oscillating electrons inside a single metallic nanoparticle (MNP), a comprehensive methodology is presented in the paper for calculating the equivalent circuit elements associated with an MNP. To find out the passive circuit elements for the MNP, the electromagnetic (EM) power flows are calculated by deriving the relaxation damping, radiation outflow, host matrix EM coupling, and applied signal interaction. The law of conservation of energy is then used to compute the extended oscillatory equation motion of a spherical MNP. The resonant behavior of a single MNP is represented by a lumped resonant circuit model, where the circuit parameters $R L C$ are derived from the equation of motion of the EDM and EM near-field energy outside the MNP. Finally, equivalent circuit of a linearly equispaced MNPs plasmon wire is modeled as a voltage-controlled voltage source by using the nearest surface plasmon interactions.
\end{abstract}

Index Terms-Full-width at half-maximum (FWHM), lumped resonant circuit model, optical interconnect, radiation damping, relaxation damping, surface plasmon (SP), surrounding matrix damping, voltage-controlled voltage source (VCVS).

\section{INTRODUCTION}

$\mathbf{T}$ HE FEASIBILITY of silicon lasing, photodetection, and cointegration of on-chip optical interconnect has sparked enormous optimism in CMOS very large-scale integration (VLSI) industry because intrachip high-speed binary data transfer via high-bandwidth optical waveguides is expected to solve various limitations of conventional metallic wires that are dominantly used in intrachip wiring in commercial chips. With aggressive device and interconnect scaling, metallic interconnect is witnessing several formidable problems, namely, increasing propagation delay on long global wires, increasing power dissipation due to wide-scale insertion of signal boosting buffers, and intrinsic coupling between wires causing signal-dependent time delays and logical faults. Optical interconnect, in contrast, offers very small interconnect delay, high bandwidth of data transfer, significantly reduces power consumption by obviating the need of repeaters, and virtually eliminates the coupling

Manuscript received October 20, 2006. First published September 19, 2008; current version published May 6, 2009. This work was supported in part by the Air Force Office of Scientific Research (AFOSR) grant. The review of this paper was arranged by Associate Editor H. Misawa.

$\mathrm{K}$. Song is with the Department of Mechanical Engineering, University of Michigan, Ann Arbor, MI 48109-2121 USA (e-mail: songk@umich.edu).

P. Mazumder is with the Department of Electrical Engineering and Computer Science, University of Michigan, Ann Arbor, MI 48109-2121 USA (e-mail: mazum@eecs.umich.edu).

Color versions of one or more of the figures in this paper are available online at http://ieeexplore.ieee.org.

Digital Object Identifier 10.1109/TNANO.2008.2005493 noise and electromagnetic (EM) interference between various adjacent wires [1].

However, light cannot propagate along the conventional waveguide if it is smaller than the wavelength of the optical signal [2]. As an alternative to solving the diffraction limit of optical signals, surface plasmon (SP) is now being extensively pursued for fabricating nanoscale photonic devices [3]. Notably, a metal has a negative dielectric permittivity in the optical spectrum, thereby enabling 1-D or 2-D SP wave to be propagated over subwavelength metallic structures [2], [4]. Especially, the selective optical absorption of a spherical-like metallic nanoparticle (MNP) allows us to develop the next-generation photonic devices such as subwavelength waveguides and SP biosensors. With the development of nanofabrication technology such as electron beam lithography (EBL) [5], the ordered metallic nanostructures can now be built to manipulate the efficient SP coupling between EM and the metallic surface [6], [7]. Some analytical calculations [7], [8] and numerical approaches [6], [9] have been developed to describe EM signal energy transfer along the MNPs. In addition, equivalent nanocircuit elements of MNP in the optical domain have been recently developed [10], [11]. These equivalent nanocircuit elements allow us to investigate future MNPs applications such as subwavelength imaging [12], quantum optics [13], nanoscale waveguide [6], [7], and nearfield optics [14]. However, these models [10], [11] have some limitations to describe damping terms such as radiation damping and host matrix coupling effects. Furthermore, applied current element from optical signal interaction has not been presented analytically.

In this paper, we focus on rigorous way to develop the equivalent circuit modeling of an MNP and an equispaced linear MNPs array based on the electric dipole moment (EDM). First, to develop the inductance and capacitance elements, the internal oscillation energy and EM near-field energy can be calculated based on the EDM. Second, to describe the resistance elements and the applied signal, four main power flows including relaxation loss, radiation outflow emission, host matrix coupling, and applied signal interaction are calculated by using the rigorous EM analysis. Third, the conservation of energy law leads to computation of the relaxation, radiation, and surrounding matrix damping frequency. Fourth, the resonant behavior of SP modes in a single MNP is represented by a lumped SP resonant circuit model. The lumped resonant circuit parameters such as capacitance, inductance, and conductance are calculated by using the equation of motion of the EDM, electric potential, and EM nearfield energy. Finally, nanoelements of an MNP are extensible to the equivalent circuit modeling for a closely equally spaced MNPs array as a voltage-controlled voltage source (VCVS) 


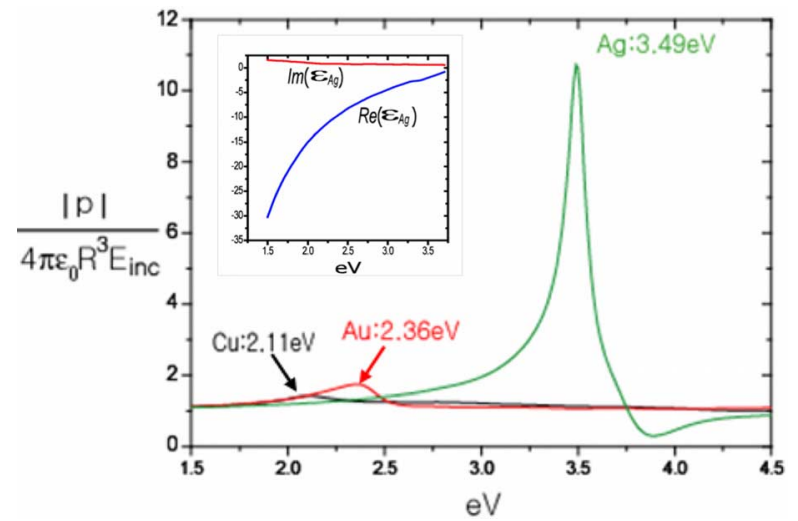

Fig. 1. Nondimensional EDM $|p| / 4 \pi \varepsilon_{0} R_{\text {out }}^{3} E_{\text {inc }}$ as a function of photon energy. These curves are obtained from the cubic spline method by using the Johnson's experimental optical data of three different noble metals: $\mathrm{Cu}, \mathrm{Au}$, and Ag.

by using the SP couplings between MNPs. Furthermore, these calculations not only give a qualitative understanding about SP modes originating from a single MNP, but also provide the physical intuition of the complex physical phenomena occurring in the MNPs waveguide.

The paper is organized as follows. Section II presents the EDM on the basis of electrostatic approximation and show the Lorentzian shape with full-width at half-maximum (FWHM) of the incident wave providing an efficient coupling. Section III outlines the internal oscillation energy and EM near-field energy of SP modes in a spherical MNP. Section IV describes the four SP power flows coming from the MNP. Section V generalizes the extended oscillatory equation of motion of the MNP and describes how radiation and surrounding matrix effect of MNP can be included in this equation. Section VI provides nanocircuit elements $(R, L, C)$ of the MNP. Finally, Section VII develops the equivalent circuit modeling for an equispaced linearly spherical MNPs plasmon wire.

\section{EDM OF A SPHERICAL MNP}

In a spherical MNP waveguide, the first spherical MNP along the chain is optically irradiated by a near-field generator, such as a near-field scanning optical microscope (NSOM), or an optical input system, such as a far- and near-field conversion structure [15], [16]. Since the wavelength of light is large compared to the geometry of particles, the validity of uniform EM field is reasonable, and the electric charge distribution produces the coherent EDM. Especially, in the nanoscale domain, SP modes generate an EM dipole field. In order to evaluate the EDM and account for strong EM absorption at the optical spectrum, we assume that uniform EM wave applies to the spherical MNP and produces the electron cloud oscillation. Under these assumptions, the EDM of the MNP can be described by [17]

$$
p=4 \pi \varepsilon_{0} R^{3}\left(\frac{\varepsilon_{r}(\omega)-1}{\varepsilon_{r}(\omega)+2}\right) E_{\text {inc }}
$$

where $R$ is the radius of an MNP and $\varepsilon_{r}(\omega)$ is the dielectric response. Fig. 1 shows the nondimensional EDM $|p| / 4 \pi \varepsilon_{0} R_{\text {out }}^{3} E_{\text {inc }}$ by using the Johnson's experimental data of three different noble metals such as $\mathrm{Cu}, \mathrm{Au}$, and $\mathrm{Ag}$ [18]. In addition, the complex dielectric constant $\varepsilon_{r}(\omega)=\varepsilon_{r e}(\omega)+$ $j \varepsilon_{i m}(\omega)$ is obtained from the cubic spline regression method, as shown in inset of Fig. 1. As can be seen, three noble metals have different magnitude of the EDM, $\mathrm{Cu}$ (1.422), $\mathrm{Au}$ (1.739), and $\mathrm{Ag}$ (10.72), with different resonant frequencies: $\mathrm{Cu}(2.11 \mathrm{eV})$, $\mathrm{Au}(2.36 \mathrm{eV})$, and $\mathrm{Ag}(3.49 \mathrm{eV})$. In general, these Lorentzian behaviors can be explained by using the Drude model approximation. The complex dielectric of noble metals can be described by the Drude model

$$
\varepsilon_{r}(\omega)=1-\frac{\omega_{p}^{2}}{\omega(\omega+j \gamma)}
$$

where $\omega_{p}=\sqrt{n_{e} e^{2} / \varepsilon_{0} m_{e}}$ is the bulk plasma frequency and $\gamma$ is the relaxation damping frequency. It follows from the previous equations that the EDM can be written as

$$
p=4 \pi \varepsilon_{0} R^{3} \frac{-\omega_{p}^{2}}{3 \omega(\omega+j \gamma)-\omega_{p}^{2}} E_{\text {inc }} .
$$

From (3), the resonant frequency can be calculated as

$$
\omega_{o}=\sqrt{\frac{\omega_{p}^{2}}{3}-\frac{\gamma^{2}}{2}} .
$$

However, in the case of the MNP, because the bulk plasma frequency $\left(\omega_{p}\right)$ is about ten times higher in magnitude than the relaxation frequency $(\gamma)$ [4], [18], the relaxation damping frequency effect on the resonance frequency is almost negligible. Therefore, the resonant frequency is almost equal to $\omega_{o}=\sqrt{1 / 3} \omega_{p}$. However, the Lorentzian line shape with FWHM depends on bulk plasma frequency $\left(\omega_{p}\right)$ and relaxation damping frequency $(\gamma)$. For instance, as shown in Fig. 1, Ag with high bulk plasma frequency $\left(\omega_{p}\right)$ and low relaxation frequency $(\gamma)$ generates the localized EM field enhancement compared to other noble metals such as $\mathrm{Au}$ and $\mathrm{Cu}$.

\section{INTERNAL OSCILLATION ENERGY AND EM NEAR-FIELD ENERGY}

To demonstrate inductance and capacitance of the MNP, it is necessary to calculate the internal oscillation energy and EM near-field energy in that internal oscillation energy gives the equation of motion of SP modes, and EM near-field energy allows us to calculate the energy of the capacitance element. First, in order to calculate the internal oscillation and EM near-field energy, we begin to examine the basic physical mechanism of SP mode in the MNP. Inside the MNP, light creates an electron gas displacement with respect to positive-ion charge. The surface charge polarization generates an electric field that acts as the restoring force inside the MNP. Thus, this harmonic oscillatory motion is analogous to simple mechanical motion, which is described by the spring constant and the relative displacement from the equilibrium position. The spring constant and resonant frequency of electron clouds can be calculated by an internal electric field inside the MNP. Accordingly, the internal oscillation energy of electron density of the MNP can be described in 
terms of the EDM

$$
H_{O}=\frac{1}{2} \frac{m_{e}}{N e^{2}}\left[\frac{d p}{d t}\right]^{2}+\frac{1}{2} \frac{m_{e} \omega_{o}^{2}}{N e^{2}} p^{2}
$$

where the first term denotes the kinetic energy, the second term describes the potential energy, $p=\int x^{\prime} \rho\left(x^{\prime}\right) d^{3} x^{\prime}$ is the EDM, $\omega_{o}$ is the resonant oscillation frequency, $N$ is the number of electrons, and $m_{e}$ is the optical electron mass. Note that $N=$ $4 \pi R^{3} \rho / 3$, where $\rho$ is the electron gas concentration and $R$ is the radius of the MNP. However, in contrast to a uniformly polarized infinite dielectric slab, the spherical surface charge distribution with axial symmetry produces the near-field EM energy outside the MNP. To calculate the near-field EM energy, we make two assumptions: 1) almost all EM energy density can be generated by the EDM because particle dimensions are small compared to the wavelength of oscillation frequency and 2) the surrounding medium is linear dispersive with small attenuation coefficient and frequency-independent permittivity. With two assumptions, the electric field in the near-field zone $(k r \ll 1)$ can be calculated by the localized oscillating system [17]

$$
E(r, t)=\frac{1}{4 \pi \varepsilon r^{3}}\left[3 \vec{e}_{r}\left(\vec{e}_{r} \cdot \vec{p}\right)-\vec{p}\right]
$$

where $\varepsilon$ is the permittivity in the surrounding matrix, $\vec{p}=$ $p(r) \cos \left(\omega_{o} t\right) \vec{e}_{z}$ is the EDM inside the MNP, and $\vec{e}_{r}$ is a unit vector in the radial direction. And, the effective EM energy can be described by the Poynting's theorem

$$
\frac{1}{2} \operatorname{Re}\left[\frac{d(\omega \varepsilon)}{d \omega}\right]\langle E(r) \cdot E(r)\rangle \approx \frac{1}{2} \varepsilon\langle E(r) \cdot E(r)\rangle .
$$

Therefore, the near-field EM energy outside the MNP can be obtained by integrating over the whole volume outside the particle

$$
E_{N}=\frac{\varepsilon}{2} \int_{R}^{\infty} \int_{0}^{2 \pi} \int_{0}^{\pi} E(r) \cdot E(r) r^{2} \sin \theta d \theta d \phi d r .
$$

The near-field EM energy can be expressed in terms of the EDM

$$
E_{N}=\frac{1}{12} \frac{p^{2}}{\pi \varepsilon R^{3}} \approx \frac{1}{24} \frac{|p|^{2}}{\pi \varepsilon R^{3}}
$$

where $|p|=\sqrt{\vec{p}^{*} \cdot \vec{p}}$ means the magnitude of the EDM. The term is responsible for dispersion relationship, optical force, and EM energy transfer along the MNP waveguide.

\section{POWER Flow CAlCUlation OF A SPHERICAl MNP}

In this section, we now focus on resistance elements and the applied current signal. The resistance elements and the applied current signal are strongly related to power flows. In the case of resistance elements, there exist dissipative power flows because of three main possible mechanisms, including electron clouds relaxation [19], radiation energy outflow [20], and resonant surrounding matrix coupling [20] in the three different domains, as shown in Fig. 2. Assuming that relaxation collision damping force is proportional to the derivative of the electric charge displacement, the damping force for electron clouds inside the

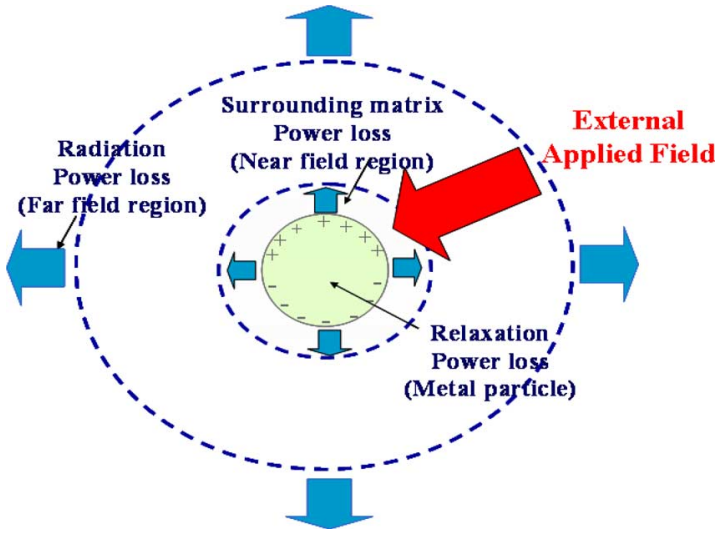

Fig. 2. For the oscillatory SP modes in an MNP, energy dissipation mechanisms such as relaxation (inside MNP), radiation energy outflow (far-field region), and surrounding matrix power loss (near-field region) are existed in three different regions.

MNP can be described by

$$
F_{D}=M_{e} \gamma\left[\frac{d x}{d t}\right]
$$

where $\gamma$ denotes the relaxation damping coefficient. Therefore, the relaxation damping power loss per unit time can be written as follows in terms of the EDM:

$$
\frac{d W_{D}}{d t}=\frac{m_{e} \gamma}{N e^{2}}\left[\frac{d p}{d t}\right]^{2} .
$$

In the case of the radiation damping loss, this damping mechanism is the emission of EM power flow outside the nanoparticle. Since the radiation pattern is similar to the dipole field distribution, radiation power depends on the dipole plasmon mode. The electric dipole field in the far-field region $(k r \gg 1)$ is given by

$$
E(r, t)=\frac{v k^{2} Z}{4 \pi}\left(\left[\vec{e}_{r} \times \vec{p}\right] \times \vec{e}_{r}\right) \frac{e^{i k r}}{r} .
$$

The Poynting vector allows us to calculate the radiation power as follows:

$$
\frac{d W_{R}}{d t}=\frac{v^{2} \mathrm{Z} k^{4}|p|^{2} \sin ^{2}\left(\omega_{o} t-r / v\right)}{6 \pi}
$$

where $v=c / n$ is the EM velocity, $Z=(\varepsilon / \mu)^{1 / 2}$ is the impedance in the surrounding matrix, and $k$ is the EM wavenumber. If we ignore the retarded time-delay phase difference $(r / v \approx 0)$ and assume the perfect harmonic oscillator model $p(r, t)=p(r) \cos \left(\omega_{o} t\right)$ because the damping decay rate is very small compared to the oscillation frequency, the radiation power loss can be approximately obtained in terms of the rate of change of momentum

$$
\frac{d W_{R}}{d t} \approx \frac{v^{2} \mathrm{Z} k^{4}}{6 \pi \omega_{o}^{2}}\left(\frac{d p}{d t}\right)^{2} .
$$

The last decay mechanism is the EM resonant coupling interaction with the surrounding matrix. In the actual dispersive nonmagnetic media such as silicon-based material, EM energy may be dissipated into the surrounding matrix. This dissipated 
power loss per unit time can be calculated as follows [17]:

$$
\frac{d}{d t}\left(W_{M}\right)=\omega_{o} \varepsilon_{0} \operatorname{Im} \chi\left(\omega_{o}\right)\langle E(r) \cdot E(r)\rangle
$$

where $\omega_{0}$ is the oscillation frequency and $\operatorname{Im} \chi\left(w_{o}\right)$ denotes the imaginary susceptibility in the surrounding matrix. Therefore, the absorbed energy in the host matrix per unit time is calculated

$$
\begin{aligned}
\frac{d}{d t}\left(W_{M}\right) & \cong \frac{\omega_{o} \varepsilon_{0} \operatorname{Im} \chi\left(\omega_{o}\right) p^{2}}{6 R^{3} \pi \varepsilon_{r e}^{2}} \\
& \cong \frac{\varepsilon_{0} \operatorname{Im} \chi\left(\omega_{o}\right)}{6 R^{3} \pi \varepsilon_{r e}^{2} \omega_{o}}\left(\frac{d p}{d t}\right)^{2} .
\end{aligned}
$$

Finally, the EM field of the applied signal interacts with electron clouds in the MNP, as shown in Fig. 2. This interaction between the applied signal and electron clouds produces the external force in the MNP

$$
F=-N e E_{I}
$$

where $E_{I}$ means the electric field of the applied signal. The power flow of the applied signal per unit time can be calculated in terms of the EDM

$$
\frac{d W_{A}}{d t}=-E_{I} \frac{d p}{d t}
$$

\section{EXTENDED OSCILLATORY EQUATION MOTION OF A SPHERICAL MNP}

To obtain the equation of motion of SP modes in a single MNP, the time derivative of internal oscillation energy in the MNP is equal to power flow due to the relaxation, radiation, surrounding matrix, and applied signal

$$
\frac{d}{d t}\left(H_{O}\right)=-\frac{d W_{D}}{d t}-\frac{d W_{R}}{d t}-\frac{d W_{M}}{d t}+\frac{d W_{A}}{d t} .
$$

The extended oscillatory equation of motion including all damping mechanisms in the MNP can be described by

$$
\ddot{p}+\left(\gamma+\gamma_{R}+\gamma_{M}\right) \dot{p}+\omega_{o}^{2} p=-\frac{N e^{2}}{m_{e}} E_{I}
$$

where $\gamma_{R}$ is the radiation damping frequency and $\gamma_{M}$ is the surrounding damping matrix. The radiation damping frequency is given by

$$
\gamma_{R}=\frac{2 \rho e^{2} v^{2} Z k^{4} R^{3}}{9 m_{e} \omega_{o}^{2}}=\left(\frac{2 \rho e^{2} Z_{0}}{9 m_{e}}\right)\left(\frac{n}{c^{2}}\right) \omega_{o}^{2} R^{3}
$$

where $Z=\left(\varepsilon_{0} / \mu\right)^{1 / 2}$ is the impedance in the air matrix. And, the matrix damping frequency is also determined by

$$
\gamma_{M}=\frac{2 \rho e^{2} \varepsilon_{0} \operatorname{Im} \chi\left(\omega_{o}\right)}{9 m_{e} \varepsilon_{\mathrm{real}}^{2} \omega_{o}}=\left(\frac{2 \rho e^{2}}{9 m_{e} n^{4} \varepsilon_{0}}\right)\left(\frac{\operatorname{Im} \chi\left(\omega_{o}\right)}{\omega_{o}}\right) .
$$

It follows from (21) and (22) that radiation damping frequency $\left(\gamma_{R}\right)$ depends on the particle size, SP oscillation frequency, and metal physical properties, whereas matrix damping frequency $\left(\gamma_{M}\right)$ relies on the optical properties of the surrounding matrix, SP oscillation frequency, and metal physical properties.

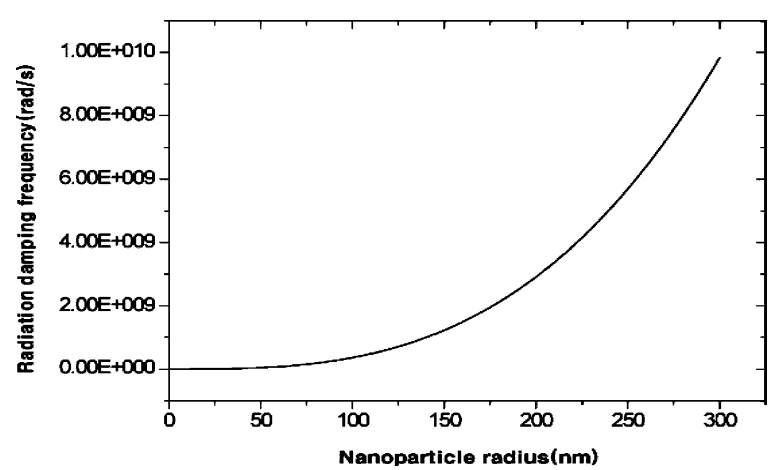

Fig. 3. Radiation damping frequency $\left(\gamma_{R}\right)$ can be calculated corresponding to different radii. This calculation is performed for $\mathrm{Ag}$.

To obtain radiation damping frequency, we choose Ag particles with different radii because the radiation frequency relies on the particle size. For this calculation, we have used electron concentration $\rho_{\mathrm{Ag}}=5.85 \times 10^{28} / \mathrm{m}^{3}$ and an electron mass $m_{\mathrm{Ag}}=8.745 \times 10^{-31} \mathrm{~kg}$ for $\mathrm{Ag}$ [21]. The surrounding matrix is chosen to be $\mathrm{SiO}_{2}$ with small attenuation coefficient $\alpha \sim 3 / \mathrm{m}$ and the refractive index $n \sim 1.56$ under the assumption of an isotropic medium in the optical regime. For maximum EDM, we use the resonant oscillation frequency $\omega_{o}=5.302 \times 10^{15} \mathrm{rad} / \mathrm{s}(3.49 \mathrm{eV})$, as shown in Fig. 1. For these parameters, we calculate the imaginary susceptibility $\operatorname{Im} \chi\left(\omega_{o}\right) \sim n \alpha c / \omega_{o}=2.546 \times 10^{-7}$ and the surrounding matrix damping frequency $\gamma_{M}=3.49 \times 10^{8} \mathrm{rad} / \mathrm{s}$. Fig. 3 shows the radiation frequency corresponding to the radius of MNP. Radiation damping frequency $\left(\gamma_{R}\right)$ is almost equal to the value of $2.91 \times 10^{9} \mathrm{rad} / \mathrm{s}$ for $200 \mathrm{~nm}$ radius. Therefore, in the case of resonant oscillation mode with small radius MNP and small attenuation coefficient of the surrounding matrix, radiation and surrounding matrix damping frequency are negligible compared to relaxation damping frequency since relaxation damping $(\gamma)$ has the order of $10^{14} \mathrm{rad} / \mathrm{s}$ [18]. However, the radiation damping $\left(\gamma_{R}\right)$ can be an important factor in the event of high oscillation frequency and large particle dimension, whereas the matrix damping frequency can be a significant factor in a poor host matrix with large attenuation coefficient.

\section{NANOCIRCUIT ELEMENTS OF AN MNP}

The SP resonant behavior of an MNP can be modeled as a lumped resonant circuit, as shown in Fig. 4. The circuit equation for the lumped resonant circuit is given by

$$
\frac{d^{2} v(t)}{d t^{2}}+\frac{G}{C} \frac{d v(t)}{d t}+\frac{1}{L C} v(t)=\frac{1}{C} \frac{d i}{d t}
$$

where $v(t)$ represents the equivalent current voltage, and $C, L$, and $G$ denote capacitance, inductance, and conductance, respectively. In the spherical MNP, the average electric potential can be written in terms of the EDM. The average electric potential around the MNP can be calculated by integration of the internal electric field. In general, the symmetric charge configuration of 


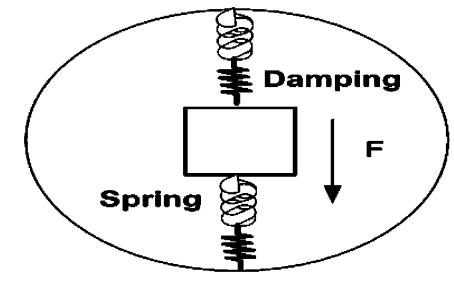

(a)

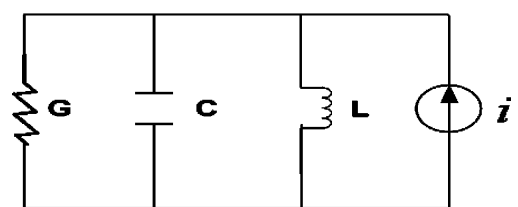

(b)

Fig. 4. Equivalent models for a plasmonic MNP. (a) Mechanical spring-massdamping model. (b) Lumped resonant circuit model.

a hemispherical region gives the average potential as follows:

$$
\begin{aligned}
v_{\mathrm{avg}} & =\operatorname{avg}\left[\int-E_{\mathrm{int}} d l\right]=-\frac{4 R}{3} E_{\mathrm{int}} \\
& =-\frac{p}{\pi R^{2}\left(\varepsilon-\varepsilon_{0}\right)}
\end{aligned}
$$

where $E_{\text {int }}=3 \varepsilon E_{I} /\left(\varepsilon+2 \varepsilon_{0}\right)$ denotes the internal electric field inside the MNP [10]. Therefore, the equation of motion in the lumped resonant circuit can be described in terms of the EDM:

$$
\frac{d^{2} p}{d t^{2}}+\frac{G}{C} \frac{d p}{d t}+\frac{1}{L C} p=\frac{-\pi R^{2}\left(\varepsilon-\varepsilon_{0}\right)}{C} \frac{d i}{d t}
$$

It has been shown that three related equations between the lumped element model and the extended equation of motions of the EDM can be established by connecting (20) and (25) as follows:

$$
\begin{aligned}
\frac{G}{C} & =\gamma+\gamma_{R}+\gamma_{M}=\gamma_{T}, \quad \frac{1}{L C}=\omega_{o}^{2}, \\
\frac{N e^{2}}{m_{e}} E_{I} & =\frac{\pi R^{2}\left(\varepsilon-\varepsilon_{0}\right)}{C} \frac{d i}{d t} .
\end{aligned}
$$

There are four lumped unknown parameters $(G, C, L, i)$ and three related equations between the lumped circuit model and the equation of motion of the MNP. Therefore, to determine all circuit parameters, it is necessary to set up one additional relation. In the optical spectrum, the metallic structures have the negative permittivity that can be described by the inductance $(L)$, and surrounding material has the positive permittivity that can be presented by the capacitance $(C)$ [10]. Therefore, the stored energy in the capacitance element in the lumped resonant circuit is almost equal to the EM near-field energy outside MNP.

$$
U_{C}=\frac{1}{2} C v_{\mathrm{avg}}^{2} \cong \frac{1}{4} C\left|v_{\mathrm{avg}}\right|^{2}=\frac{1}{24} \frac{|p|^{2}}{\pi \varepsilon_{0} R^{3}} .
$$

The capacitance of an MNP is given by

$$
C=\frac{\pi R\left|\varepsilon-\varepsilon_{0}\right|^{2}}{6 \varepsilon_{0}} .
$$

Table I shows $C, L$, and $G$ components for three different noble MNP (radius $20 \mathrm{~nm}$ ) at three different resonant frequencies,
TABLE I

NANOCIRCUIT ELEMENTS OF THREE NOBLE MNPS (RADIUS $R=20 \mathrm{NM}$ ) That IS SURROUNDED By a FrEe SPACE WITH A REFRACTIVE INDEX $n=1$

\begin{tabular}{|c|c|c|c|}
\hline & $\mathrm{Cu}$ & $\mathrm{Au}$ & $\mathrm{Ag}$ \\
\hline $\begin{array}{c}\mathrm{eV} \\
\text { (resonance) }\end{array}$ & 2.11 & 2.36 & 3.49 \\
\hline $\begin{array}{c}\omega \circ \\
(\mathrm{Rad} / \mathrm{s})\end{array}$ & $3.206 \mathrm{e} 15$ & $3.585 \mathrm{e} 15$ & $5.302 \mathrm{e} 15$ \\
\hline $\begin{array}{c}\varepsilon \\
\left(1 / \varepsilon_{0}\right)\end{array}$ & $-7.262+\mathrm{j} 3.460$ & $-4.261+\mathrm{j} 2.435$ & $-2.068+\mathrm{j} 0.279$ \\
\hline$\gamma$ & $1.449 \mathrm{e} 14$ & $1.075 \mathrm{e} 14$ & $3.226 \mathrm{e} 13$ \\
\hline $\mathrm{C}$ & $7.439 \mathrm{af}$ & $3.124 \mathrm{af}$ & $0.880 \mathrm{af}$ \\
\hline $\mathrm{L}$ & $13.08 \mathrm{fF}$ & $24.91 \mathrm{fF}$ & $40.43 \mathrm{fF}$ \\
\hline $\mathrm{G}$ & $1.078 \mathrm{mS}$ & $0.339 \mathrm{mS}$ & $0.0283 \mathrm{mS}$ \\
\hline
\end{tabular}

(a)

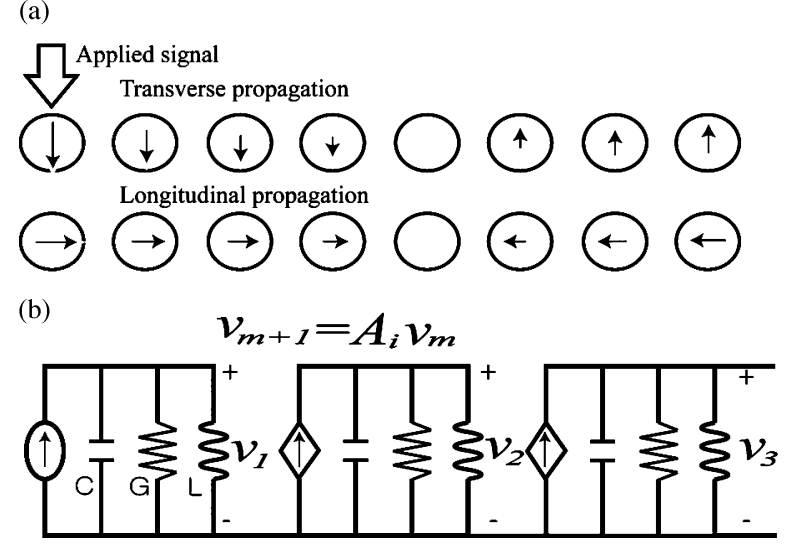

Fig. 5. (a) Two fundamental coupled oscillations of a plasmon wire: longitudinal oscillations and transverse oscillation. (b) Equivalent circuit modeling for MNPS plasmon wire as a VCVS.

which is surrounded by a free space with a refractive index $n=1$. To calculate nanocircuit components, the relaxation damping frequency $(\gamma)$ is taken from the Johnson's data [18]. As can be seen, compared with $\mathrm{Cu}$ and $\mathrm{Au}, \mathrm{Ag}$ has high inductance and small conductance for efficiently sending SP along the MNPs. At resonant frequency $\omega_{o}=5.302 \times 10^{15} \mathrm{rad} / \mathrm{s}$, the permittivity of $\mathrm{Ag}$ has the value of $(-2.068+j 0.279) \varepsilon_{0}$ obtained by Johnson's experimental optical data [18]. Let us assume the spherical MNP with $R=20 \mathrm{~nm}$. Equations (26) and (28) show that the circuit parameters of Ag of the MNP has the value of $C=0.880 \mathrm{aF}, L=40.43 \mathrm{fH}$, and $G=0.0283 \mathrm{mS}$.

\section{EQUivalent CiRcuit of MNPS Plasmon WIRE}

The subwavelength energy transfer along a closely equispaced MNPs plasmon wire with spatial distance $d$ can be modeled by using the nanoelements of the MNP. As can be seen in Fig. 5, the first MNP of the plasmon wire is illuminated by a laser, thus coupled SP modes can be transported along the MNPs array. From the point of the antenna theory, near field produced by $m$ th dipole antenna is received by identical $(m-1)$ th and $(m+1)$ th dipole antennas respectively; near field from $(m+1)$ th dipole accordingly transmits $m$ th and $(m+2)$ th dipole antennas. In addition, the efficiency of receiving antenna is also determined by the orientation between the 
receiving dipole antenna and transmitting dipole antenna. The fundamental orientations of dipole antenna in Fig. 5(a) indicate that there are two kinds of SP coupled modes: longitudinal coupled modes where dipole moments are along the propagation direction and transverse coupled modes where dipole moments are perpendicular to the axis of the array. Depending on the polarization orientation, the E-field received by the $m$ th dipole antenna from the nearest SP coupling is given by using (6):

$$
E_{p, m}=\frac{\mu_{i} p_{m+1}(t)}{4 \pi \varepsilon_{0} n^{3} d^{3}}+\frac{\mu_{i} p_{m-1}(t)}{4 \pi \varepsilon_{0} n^{3} d^{3}}
$$

where $\mu_{i}$ is the polarization constant for which $\mu_{L}=-2$ and $\mu_{T}=1, p_{m}$ is the EDM of the $m$ th MNP, $n$ is the refractive index of the matrix, and $d$ is the distance of an equally spaced linear array. The equation of motions of the $m$ th $(m \geq 2)$ MNP can be obtained by using the nearest EDM interaction

$$
\ddot{p}_{i, m}+\gamma_{T} \dot{p}_{i, m}+\omega_{o}^{2} p_{i, m}=-\mu_{i} \omega_{c}^{2}\left(p_{i, m-1}+p_{i, m+1}\right)
$$

where the SP coupling frequency will be

$$
\omega_{c}^{2}=\frac{N e^{2}}{4 \pi \varepsilon_{0} n^{3} d^{3} m_{e}}=\frac{\rho R^{3} e^{2}}{3 \varepsilon_{0} n^{3} d^{3} m_{e}} s .
$$

The propagating wave solution to (30) have the following form [7]:

$$
p_{i, m}=p_{i, m-1} \exp (-\alpha d+j(\omega t \pm k d))
$$

where $p_{m-1}$ and $p_{m}$ are the EDM of $(m-1)$ th and $m$ th MNP, respectively, and $\alpha$ is the damping constant per unit length. Substituting (32) into (30) yields

$$
\begin{aligned}
& \omega \gamma_{T}+2 \mu_{i} \omega_{c}^{2} \sin (k d) \sinh (\alpha d)=0 \\
& \omega^{2}=\omega_{o}^{2}+2 \mu_{i} \omega_{c}^{2} \cos (k d) \cosh (\alpha d) .
\end{aligned}
$$

For $\alpha d \ll 1$, attenuation constant is approximately given by

$$
\alpha_{i}=\frac{\gamma_{T} \omega}{2\left|\mu_{i}\right| d \sin (k d) \omega_{c}^{2}}
$$

where $\alpha_{T}$ and $\alpha_{L}$ are the transverse and longitudinal damping factor, respectively. At the resonance frequency $\left(\omega=\omega_{0}\right)$, the wavenumber condition should be satisfied:

$$
k d=\frac{\pi}{2} \text {. }
$$

Since the voltage of the $m$ th MNP can be calculated by the EDM of the $m$ th MNP

$$
v_{m}(t)=-\frac{p_{m}(t)}{\pi R^{2}\left(\varepsilon-\varepsilon_{0}\right)} .
$$

The parameter that relates $v_{m}$ and $v_{m-1}$ is the MNP plamson wire $A_{i}=v_{m} / v_{m-1}$

$$
A_{i}=\exp \left(-\alpha_{i} d\right) \exp ( \pm j k d) .
$$

Fig. 5(b) shows the equivalent circuit model for the closely linear MNPs plsmon wire as a VCVS with voltage gain $A_{i}$. Thus, voltage gain $\left(A_{i}\right)$ has magnitude

$$
\left|\frac{A_{m}}{A_{m-1}}\right|=\exp \left(-\alpha_{i} d\right) .
$$

Table II gives the equivalent circuit parameters including cou-
TABLE II

Equivalent Circuit Parameters of Three Noble MNPS Plasmon Wire (RADIUS $R=20 \mathrm{NM}$ ) SEPARATED By a SPATIAL DiSTANCE $(d=60 \mathrm{NM})$ AT THREE DiFFERENT RESONANT FREQUENCIES, Which IS SURROUNDED BY A FREE SPACE With a REFRACTIVE INDEX $n=1$

\begin{tabular}{|c|c|c|c|}
\hline & $\mathrm{Cu}$ & $\mathrm{Au}$ & $\mathrm{Ag}$ \\
\hline $\begin{array}{c}\mathrm{eV} \\
\text { (resonance) }\end{array}$ & 2.11 & 2.36 & 3.49 \\
\hline $\begin{array}{c}\omega_{\mathrm{o}} \\
\text { (Rad/s) }\end{array}$ & $3.206 \mathrm{e} 15$ & $3.585 \mathrm{e} 15$ & $5.302 \mathrm{e} 15$ \\
\hline $\begin{array}{c}m \\
\text { (electron mass) }\end{array}$ & $-7.262+\mathrm{j} 3.460$ & $-4.261+\mathrm{j} 2.435$ & $-2.068+\mathrm{j} 0.279$ \\
\hline $\begin{array}{c}\rho \\
\text { (electron density) }\end{array}$ & $8.45 \mathrm{e} 28 / \mathrm{m}^{3}$ & $5.90 \mathrm{e} 28 / \mathrm{m}^{3}$ & $5.85 \mathrm{e} 28 / \mathrm{m}^{3}$ \\
\hline $\begin{array}{c}\omega_{c} \\
\text { (coupling frequency) }\end{array}$ & $1.508 \mathrm{e} 15$ & $1.530 \mathrm{e} 15$ & $1.547 \mathrm{e} 15$ \\
\hline $\begin{array}{c}\alpha_{T} \\
\text { (Transverse damping) }\end{array}$ & $1.702 \mathrm{e} 6 / \mathrm{m}$ & $1.379 \mathrm{e} 6 / \mathrm{m}$ & $5.956 \mathrm{e} 5 / m$ \\
\hline $\begin{array}{c}\alpha_{L} \\
\text { (Longitudinal damping }\end{array}$ & $8.512 \mathrm{e} 5 / m$ & $6.859 \mathrm{e} 5 / m$ & $2.978 \mathrm{e} 5 / m$ \\
\hline exp(- $\left.\alpha_{T} \mathrm{~d}\right)$ & 0.9209 & 0.9631 & 0.9649 \\
\hline $\exp \left(-\alpha_{L} \mathrm{~d}\right)$ & 0.9502 & 0.9597 & 0.9823 \\
\hline
\end{tabular}

(a)

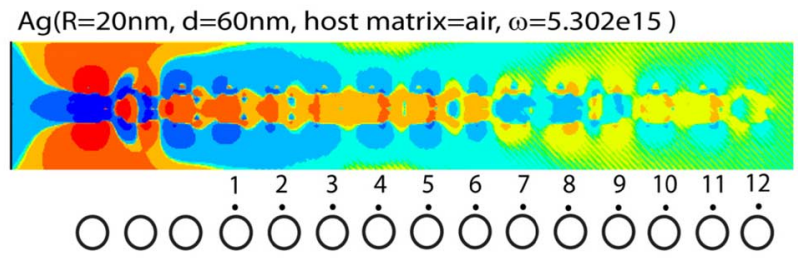

(b)
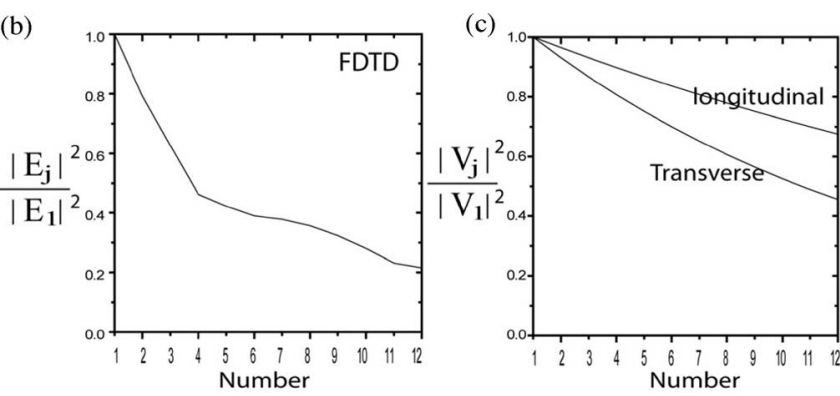

Fig. 6. (a) Time snapshots of $z$ component (in the $x y$ plane) of scattered electric field in the Ag plasmon wire. (b) Average E-field density in the Ag MNPs plasmon wire is obtained by FDTD simulation. Values are calculated at the identical positions of each MNP, and E-field densities are normalized to the E-field density of position 1. (c) Average voltage of the Ag plasmon wire is calculated by $\mathrm{Ag}$ circuit parameters. The voltages of MNPs plasmon wire at different positions are normalized to the voltage of position 1 .

pling frequency $\omega_{c}$, attenuation factor $\alpha$, voltage gain $\exp (-\alpha \mathrm{d})$ for three different noble MNPs' (radius $R=20 \mathrm{~nm}$ ) array with the spatial distance $(d=60 \mathrm{~nm})$ at three different resonant frequencies, which is surrounded by a free space with a refractive index $n=1$. To demonstrate the validity of our equivalent circuit parameters, we have conducted 3-D scattered finite-difference time-domain (FDTD) simulation. Fig. 6(a) plots the EM energy transfer in Ag plasmon wire with radius $20 \mathrm{~nm}$ and distance $60 \mathrm{~nm}$. For realizing a resonant SP subwavelength transfer along the plasmon wire, the incident plane wave ( $\omega=5.302 \times 10^{15} \mathrm{rad} / \mathrm{s}$ ), polarized parallel to the longitudinal axis of the wire, irradiates at the first MNP. Accordingly, we obtain the average E-field density at different locations from position 1 to position 12 . Values are calculated at the identical 
positions located at the $30 \mathrm{~nm}$ offset points ( $z$-directed) from the center of MNPs, and E-field densities are normalized to the E-field density of position 1. In addition, Fig. 6(c) shows the voltage attenuation based on Table II. As shown in Fig. 6(b) and (c), the FDTD calculation, from position 1 to position 3, provides strong damping coefficient compared to equivalent circuit modeling because we ignore higher order electric moments with small SP interaction compared to EDM coupling. However, FDTD simulation, after position 3, shows that attenuation coefficient is closely similar to the voltage attenuation since the SP energy transfer along the MNPs plasmon wire is transported by strong EDM coupling, as plotted in Fig. 6(a), thus validating our equivalent circuit modeling.

\section{CONCLUSION}

To calculate the MNP nanocircuit elements, the theoretical SP physical mechanism of the single MNP has been demonstrated. As described in previous sections, the spherical MNP has a lot of advantages over any other plasmonic structures in that they demonstrate optical selectivity (FWHM), an SP modes resonator, and an effective near-field EM generator. Furthermore, instead of full-wave computation-intensive FDTD simulation, the lumped element circuit model of the MNPs plasmon wire offers us fast system-level simulation models such as SPICE to investigate the behavior of the SP modes in MNPs in the presence of conventional electronic components. Therefore, we can efficiently analyze complex MNPs structure combined with photonic and electric elements by using the equivalent circuit modeling.

\section{REFERENCES}

[1] D. A. B. Miller, "Rationale and challenges for optical interconnects to electronic chips," Proc. IEEE, vol. 88, no. 6, pp. 728-749, Jun. 2000.

[2] J. Takahara, S. Yamagishi, H. Taki, A. Morimoto, and T. Kobayashi, "Guiding of a one-dimensional optical beam with nanometer diameter," Opt. Lett., vol. 22, pp. 475-477, 1997.

[3] W. A. Challener, I. K. Sendur, and C. Peng, "Scattered field formulation of finite difference time domain for a focused light beam in dense media with lossy materials," Opt. Exp., vol. 11, pp. 3160-3170, 2003.

[4] U. Kreibig and M. Vollmer, "Optical properties of metal clusters," in Optical Properties of Metal Clusters. Berlin, Germany: SpringerVerlag, 1994.

[5] W. Srituravanich, N. Fang, C. Sun, Q. Luo, and X. Zhang, "Plasmonic nanolithography," Nano Lett., vol. 4, pp. 1085-1088, 2004.

[6] M. Quinten, A. Leitner, J. R. Krenn, and F. R. Aussenegg, "Electromagnetic energy transport via linear chains of silver nanoparticles," Opt. Lett., vol. 23, pp. 1331-1333, 1998.

[7] M. L. Brongersma, J. W. Hartman, and H. A. Atwater, "Electromagnetic energy transfer and switching in nanoparticle chain arrays below the diffraction limit," Phys. Rev. B, vol. 62, pp. R16356-R16359, 2000.

[8] S. Y. Park and D. Stroud, "Surface-plasmon dispersion relations in chains of metallic nanoparticles: An exact quasistatic calculation," Phys. Rev. B, vol. 69, no. 12, pp. 125418.1-125418.7, 2004.

[9] S. A. Maier, P. G. Kik, and H. A. Atwater, "Optical pulse propagation in metal nanoparticle chain waveguides," Phys. Rev. B, vol. 67, pp. 2054021-205402-3, 2003.

[10] N. Engheta, A. Salandrino, and A. Alu, "Circuit elements at optical frequencies: Nanoinductors, nanocapacitors, and nanoresistors," Phys. Rev. Lett., vol. 95, no. 9, pp. 095504-1-095504-4, 2005.

[11] M. Alam and Y. Massoud, "RLC ladder model for scattering in single metallic nanoparticles," IEEE Trans. Nanotechnol., vol. 5, no. 5, pp. 491498, Sep. 2006.
[12] A. Ono, J. Kato, and S. Kawata, "Subwavelength optical imaging through a metallic nanorod array," Phys. Rev. Lett., vol. 95, no. 26, pp. 267407267410, 2005.

[13] D. E. Chang, A. S. Sorensen, P. R. Hemmer, and M. D. Lukin, "Quantum optics with surface plasmons," Phys. Rev. Lett., vol. 97, no. 5, pp. 0530021-053002-4, 2006.

[14] W. L. Barnes, A. Dereux, and T. W. Ebbesen, "Surface plasmon subwavelength optics," Nature, vol. 424, pp. 824-830, 2003.

[15] W. Nomura, M. Ohtsu, and T. Yatsui, "Nanodot coupler with a surface plasmon polariton condenser for optical far/near-field conversion," Appl. Phys. Lett., vol. 86, no. 18, pp. 181108-1-181108-3, 2003

[16] L. L. Yin, V. K. Vlasko-Vlasov, J. Pearson, J. M. Hiller, J. Hua, U. Welp, D. E. Brown, and C. W. Kimball, "Subwavelength focusing and guiding of surface plasmons," Nano Lett., vol. 5, pp. 1399-1402, 2005.

[17] J. D. Jackson, "Classical electrodynamics," in Classical Electrodynamics. New York: Wiley, 1999.

[18] P. B. Johnson and R. W. Christy, "Optical-constants of noble-metals," Phys. Rev. B, vol. 6, pp. 4370-4379, 1972.

[19] E. J. Heilweil and R. M. Hochstrasser, "Nonlinear spectroscopy and picosecond transient grating study of colloidal gold," J. Chem. Phys., vol. 82, pp. 4762-4770, 1985.

[20] A. Melikyan and H. Minassian, "On surface plasmon damping in metallic nanoparticles," Appl. Phys. B-Lasers Opt., vol. 78, pp. 453-455, 2004.

[21] C. Kittel, "Introduction to solid state physics," in Introduction to Solid State Physics. New York: Wiley, 1986.

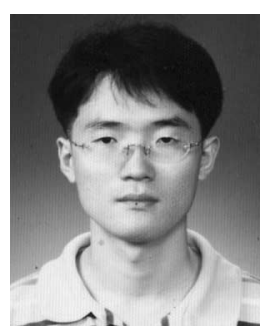

Kyungjun Song received the B.S. degree in mechanical engineering from Seoul National University, Seoul, Korea, and the M.S. degree in mechanical engineering from the University of Michigan, Ann Arbor, in 2002 and 2004, respectively. He is currently working toward the Ph.D. degree in mechanical engineering at the University of Michigan.

He is a Graduate Student Research Assistant in the Electrical Engineering and Computer Science Department, University of Michigan. He is currently engaged in research on terahertz $(\mathrm{THz})$ spoof surface plasmon circuitry. His current research interests include the modeling, simulation, and design of plasmonic nanoarchitecture.

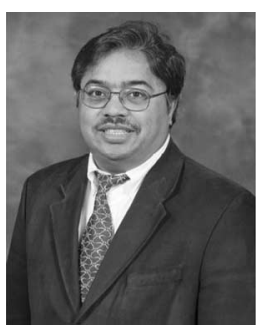

Pinaki Mazumder (F'99) received the Ph.D. degree from the University of Illinois at Urbana-Champaign, Urbana-Champaign, in 1988.

Currently, he is a Professor in the Department of Electrical Engineering and Computer Science, University of Michigan (UM), Ann Arbor. He is on leave for one year from the UM to serve as the lead Program Director of the Emerging Models and Technologies Program at the US National Science Foundation. He was for six years with industrial R\&D centers that included AT\&T Bell Laboratories, where in 1985, he started the CONES Project- the first C modeling-based VLSI synthesis tool at India's premier electronics company, Bharat Electronics Ltd., where he had developed several high-speed and high-voltage analog integrated circuits intended for consumer electronics products. He is the author or couauthor of more than 200 technical papers and four books on various aspects of VLSI research works. His current research interests include current problems in nanoscale CMOS very large-scale integration (VLSI) design, computer-aided desgin (CAD) tools and circuit designs for emerging technologies including quantum MOS and resonant tunneling devices, semiconductor memory systems, and physical synthesis of VLSI chips.

Prof. Mazumder is an American Association for the Advancement of Science (AAAS) Fellow (2008). He was a recipient of the Digital's Incentives for Excellence Award, BF Goodrich National Collegiate Invention Award, and Defense Advanced Research Projects Agency (DARPA) Research Excellence Award. 